\title{
A Novel Energy Efficient Routing Algorithm for Hierarchically Clustered Wireless Sensor Networks
}

\author{
Huang Lu and Jie Li \\ Department of Computer Science \\ University of Tsukuba \\ Tsukuba Science City, Japan
}

\author{
Guojun Wang \\ Department of Computer Science and Technology \\ Central South University \\ Changsha, China
}

\begin{abstract}
In wireless sensor networks (WSNs), gathering sensed information, transforming the information data to the base station in an energy efficient manner, and lengthening the network lifetime are important issues. Clustering is an energy efficient way that divides sensor nodes into many clusters, each of which has a cluster-head. The routing from cluster-head sensor nodes to the base station, however, has not been well studied. In this paper, we study the routing problems for WSNs. Specifically, we propose a novel energy efficient cluster-based routing algorithm for hierarchical WSNs, in which we hierarchicalize sensor nodes into different levels using the hop number of transmissions to the base station. Cluster-head sensor nodes are selected autonomously and transmit data to the base station using multi-hop transmissions, whereas non-cluster-head sensor nodes communicate with cluster-head sensor nodes directly. We have conducted comprehensive simulation experiments for the performance evaluation. The simulation results show that, for large scale WSNs, the proposed algorithm performs better in terms of network lifetime. Moreover, the algorithm mitigates the effect of self-induced black hole and balance the energy usage in the network by employing alternative sensor nodes.
\end{abstract}

Keywords-wireless sensor networks, hierarchical clustering, routing algorithm, alternative sensor nodes.

\section{INTRODUCTION}

A wireless sensor network (WSN) is a wireless network consisting of spatially distributed devices using sensor nodes to monitor physical or environmental conditions, such as temperature, sound, and motion, at different locations. In a wireless sensor network, the individual nodes are capable of sensing their environments, processing the information locally, and sending the information to one or more collection points through a wireless link [1]. Each node has a short-range transmission due to low RF (Radio Frequency) transmit power, and the short-range transmission helps in prolonging the lifetime of the battery [2]. Many sensor systems are deployed in harsh, unattended and often adversarial physical environments such as battle fields and desert. Sensor nodes are severely constrained by the amount of battery power, which limits the lifetime and quality of the network. This is crucial for WSNs to maximize nodes' lifetimes, reduce bandwidth consumption by using local collaboration among sensor nodes $|3|[4 \mid$.

Grouping sensor nodes into clusters has been widely pursued in order to achieve the network scalability objective
[4]|5]. Every cluster would have a leader, often referred to as the cluster-head $(\mathrm{CH})$. The cluster membership may be fixed or variable. Of the benefit, clustering may conserve communication bandwidth since it limits the scope of intercluster interactions to $\mathrm{CHs}$ and avoids redundant exchange of messages among sensor nodes. Moreover, a $\mathrm{CH}$ aggregates the data collected by the sensors in its cluster and thus decrease the number of relayed packets.

In this paper, a power efficient routing algorithm for hierarchically clustered WSNs is proposed. In our proposal, we hierarchicalize sensor nodes into different levels using the hop number of transmissions to the BS. CHs are selected autonomously and communicate with the base station using multi-hop transmissions which employ the multi-hop planar model, whereas non- $\mathrm{CH}$ sensor nodes communicate with $\mathrm{CH}$ sensor nodes directly. In this model, both $\mathrm{CH}$ sensor nodes and non- $\mathrm{CH}$ sensor nodes could act as the intermediary node for data forwarding in routing.

In section II, we provide an overview of related work and issues. In section III, we describe the network model of a large scale WSN, and the mechanism of the system. In section IV, we propose the power efficient routing algorithm for hierarchical cluster-based WSNs. In section V, we analyze the routing algorithm with simulation. Section VI offers conclusion and points of future work.

\section{RELATED WORK}

In this section, an overview of related work is provided, including clustering algorithms, multi-hop data transmission, and energy hole issues.

Heinzelman, et al. [6] presented the LEACH (LowEnergy Adaptive Clustering Hierarchy) protocol for WSNs of cluster-based architecture, which is a widely known and elegant clustering algorithm, by selecting the $\mathrm{CHs}$ in rounds. LEACH achieved improvement compared to direct transmissions, as measured in terms of nodes' lifetime. Following the idea of LEACH algorithm, a number of algorithms have been presented, e.g., PEGASIS (Power-Efficient Gathering in Sensor Information Systems) [7], which use the similar concept with LEACH. In this paper, for convenience, we call this kind of cluster-based routing algorithms as LEACH-like algorithms. The routing issue, however has not been well studied in LEACH-like algorithms. Because non-CH sensor 
nodes in a cluster use the $\mathrm{CHs}$ as the intermediary node to the base station (BS), but for $\mathrm{CH}$ sensor nodes, there is no certain routing algorithm for them to communicate with the BS. Also in LEACH-like algorithms, it is assumed that all sensor nodes could communicate with the BS using large transmission power. It is unachievable in the real world of large range WSNs, because of the constraints of radio transmit equipments on sensor nodes $|2||5|$.

In cluster-based WSNs, multi-hop data transmission is used for data transmission between the $\mathrm{CHs}$ to the BS, where the direct communication between the $\mathrm{CH}$ sensor nodes and the BS is not possible due to the distance or obstacles between them. The solutions to this issue could be grouped into the following two methods [8]. One is the multi-hop planar method, in which a $\mathrm{CH}$ transmits data to the BS by forwarding its data to one of its neighbors, using existing routing algorithms, e.g., AODV (Ad-hoc Ondemand Distance Vector) [9]. Another one is the clusterbased hierarchical method, in which the network is broken into clustered layers, and the data travels simply from a lower cluster-head to a higher one in turn to the BS $|10|$.

Meanwhile, in WSNs, data gathering using multi-hop transmissions usually causes a problem to the sensor nodes which are close to the BS that, because of acting as intermediaries for data transmission, their energy would exhaust faster. It is called self-induced black hole problem or energy hole problem, which has been mentioned in many articles [8||11||12|. This problem would cause a transmission blank space around the BS, and if the blank space is too wide for sensor nodes' transmission, the WSN would lose its ability. As the methods to solve this issue, some applys the nonuniform distribution of the sensor nodes [13|, some applys routing algorithms [14]. We proposed an approach using alternative sensor nodes (ASN) in routing, to reduce the effect of the self-induced black hole problem in multihop data gathering transmission. Our approach addressing this issue is first to introduce the state of a sensor node. Then, we introduce our method to turn particular sensor nodes into ASNs. Although our approach could not solve the issue completely, it could mitigate the effect caused by that, prolong the lifetime of WSNs, also prevent the sudden death of a sensor node.

\section{Network MOdel}

We consider the network model of a large scale WSN, which consist of hundreds or thousands of sensor nodes, and be distributed in a large area. Since the sensor nodes which are far away from the BS could not communicate with the BS directly in the transmission range, all sensor nodes could reach the BS using multi-hop communications. For simplifications, it is considered a WSN system in which the data being sensed by sensor nodes is transmitted to a base station, and there is only one base station with sufficient energy near part of the sensor nodes. Because sensor nodes first transmit sensed data to the $\mathrm{CHs}$, so the $\mathrm{CH}$ sensor nodes perform data fusion. In addition, sensor nodes are desired to be designed as cheap and high energy-efficient as possible, it is assumed that all sensor nodes and the BS use symmetric radio channel with the same transmission range in the network. They are distributed randomly and densely, and their energy is constrained.

In this network model, the energy consumption patterns mainly include the data processing and transmission. Data transmission cost is generally more expensive than data processing. Thus the method that the intermediate node (e.g. a $\mathrm{CH}$ ) integrates data and sends it to the BS is preferred than the method that each sensor node directly sends data to the BS [5]. A sensor node is standing by for energy saving when it does not sense or transmit data. The operation of the mechanism is divided by rounds as shown in Figure 1, and each round includes set-up phase for constructing clusters and steady-state for transmitting data from sensor nodes to the BS via CHs. In each round, the algorithm elects $\mathrm{CHs}$ in the set-up part, and in each frame, a sensor node transmits the data to its $\mathrm{CH}$.

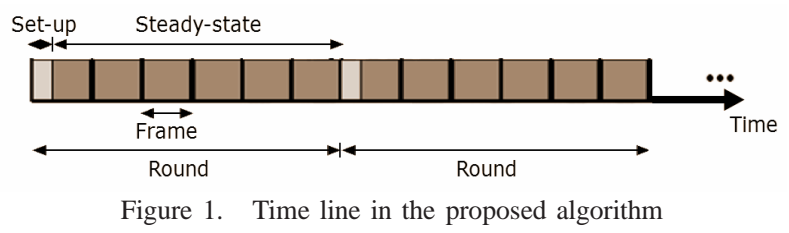

In order to increase the energy efficiency and balance the energy consumption by routing in clustered WSNs, a typical dynamic clustering method with Low-Energy Adaptive Clustering Hierarchy (LEACH) $|6|$ is used to all sensor nodes. For balancing energy consumption, each node is randomly elected as a $\mathrm{CH}$ in rounds. With $\mathrm{CHs}$, other non- $\mathrm{CH}$ sensor nodes join with clusters using one-hop transmissions, and use TDMA (Time-Division Multiple Access) for data transmission, which is scheduled by CHs. In this method, $\mathrm{CHs}$ are randomly selected in rounds. In order to select $\mathrm{CHs}$ in a round, each sensor node determines a random value and compares it with a threshold. If the value is less than the threshold, the node becomes a $\mathrm{CH}$ for the current round.

The sensor nodes in a WSN are classified into four main states: sensing only, relaying only, sensing-relaying, and inactivity [15]. In the sensing state, the node sensing circuitry is on and it sends data toward the gateway sensor node or the BS. In the relaying state, the node relays the data from other active nodes. When a node is both sensing the target and relaying messages from other nodes, it is considered in the sensing-relaying state. Otherwise, the node is considered inactive and can turn off its sensing and communication circuitry. In hierarchical cluster-based architecture, the $\mathrm{CH}$ in a cluster could be in the states of relaying only and sensing- relaying. Whereas the non-CH sensor nodes can be in all states of the four main states.

\section{Proposed Routing Algorithm}

In this section, we first describe the proposed ASN mechanism, and then present the operation details in the proposed routing algorithm. 


\section{A. Alternative Sensor Node}

In this paper, we use routing algorithm for selecting the transmission routes. There could be sensor nodes with high energy near the transmission routes. To reduce the possibility of the occurrence to the self-induced black hole problem, we introduce the mechanism of the alternative sensor node (ASN) for a sensor node in its transmission range as follows.

- Suppose that node $C$ transmits with node $D$ via node $A$, and node $B$ is in the transmission range of $A, C$, and $D$.

- Determine a distance threshold $S$ for alternative sensing range threshold.

The threshold for ASN operation should neither be too small nor too large. Suppose that, if the threshold is determined large, the routing would lost the ability of communicating with the sensor nodes on the opposite side of the original route. Meanwhile, if the distance threshold is determined small, the ASN operation would lose many opportunities of seeking the ASN sensor node. Thus, we first decide the distance threshold for the ASN operation as $S=\frac{R}{2}$ for the moment, where $R$ is the transmission range for a sensor node, and then we will optimize the value of $S$ in the future.

- Assume that node $A$ finds out node $B$ in its transmission range, and the distance between two sensor nodes $A$ and $B$ is $S_{A B}$, shown in Figure 2

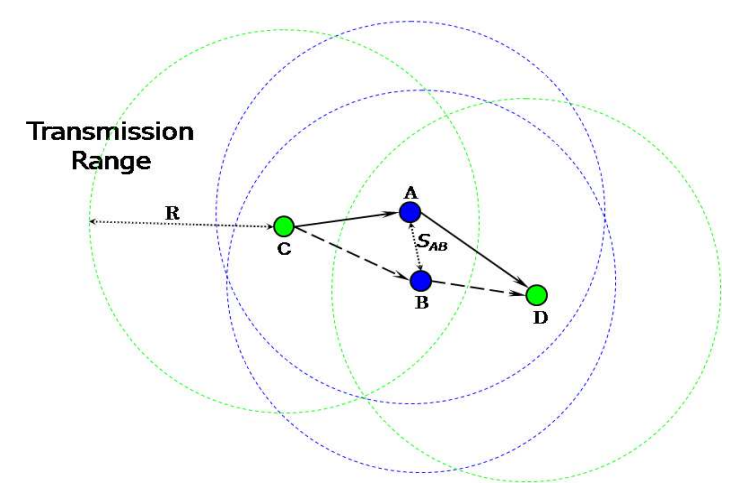

Figure 2. Alternative sensor node of node $A$

- If $S_{A B}<S$, and $E_{B}>E_{A}$, the node $B$ becomes the ASN of node $A$. Thus the transmission route from $C$ to $D$ is changed from $C \rightarrow A \rightarrow D$ into $C \rightarrow B \rightarrow D$.

In this work, the ASN mechanism is done using broadcasting by each sensor node to its neighbor nodes. From Figure 2 we can see that, after node $A$ broadcasts message to all the neighbor nodes in its transmission range, the node $B$ which fits the requirements of ASN function, returns a message to node $A, C$ and $D$, thus the new route is formed. In this method, a sensor node changes the current state into others depending on the ASN operation addressed above.

\section{B. Algorithm}

The operation of the proposed algorithm is broken up into rounds as the round changing mechanism described in section III. In each round, the BS uses broadcasting to count hop numbers and hierarchicalizes sensor nodes. Then we cluster sensor nodes into clusters, and select transmission routes for data transmission to the BS. Overall, the algorithm is divided into following five steps for conducting the data transmission in one round.

1) Counting hop number: In this step, broadcasting from the BS is used to count the hop number for each sensor node to the BS. Broadcasting is used, because the same broadcasting message from the BS could reach a sensor node through many routes. When the broadcasting messages are received by a sensor node, the sensor node uses a routing table in its memory to cache all the routes from the BS to itself.

2) Hierarchicalizing sensor nodes: Since we want to hierarchicalize sensor nodes into different layers to set the level of sensor nodes, we do it by using the hop number from the BS to each sensor node. Set sensor nodes which communicate with the BS via the same hop number into one layer. In Figure 3, we show an image that the sensor nodes are organized into hierarchical architecture.

3) Clustering in the system: In this step, the sensor nodes are clustered into clusters, and here are the points as follow.

- Use the concept of LEACH Algorithm [6] to decide cluster-head $(\mathrm{CH})$ nodes in the system, shown in Figure 3. As we addressed in section II, since each sensor node has selected a value within 0 and 1 at the beginning of the round, it compares its selected value with a threshold $T(n)$, and decide whether to become the $\mathrm{CH}$ or not. If a sensor node becomes a $\mathrm{CH}$ node independently, then it announces that to the sensor node near itself by 1-hop transmission. The threshold $T(n)$ is set as follows:

$$
\begin{array}{lll}
T(n)=\frac{P}{1-P \times\left(\operatorname{rmod}\left\lfloor\frac{1}{P}\right\rfloor\right)} & \forall n \in G & \cdots \\
T(n)=0 & \forall n \notin G & \cdots
\end{array}
$$

where $T(n)$ is the threshold for node $n$ to decide whether to turn into a $\mathrm{CH}$ with the value from 0 to 1. $P$ is a priori determined value that represents the desired percentage of $\mathrm{CH}$ nodes during a round (e.g. $P=10 \%), r$ is the round number of the current round, and $G$ is the set of nodes that have not been $\mathrm{CH}$ nodes in the last $\left\lfloor\frac{1}{P}\right\rfloor$ rounds.

- For recognize each $\mathrm{CH}$ node in their layers, we could mark them as:

Level1 $\mathrm{CH}_{1}$, Level $1 \mathrm{CH}_{2}, \ldots$ Level $2 \mathrm{CH}_{1}$, Level2 $\mathrm{CH}_{2}, \ldots$

- The other non-CH nodes join a cluster using 1 hop communication, depending on the strongest transmission signal. 
- Because there could be sensor nodes that could not find a $\mathrm{CH}$ node in their transmission range, so they elect themselves as $\mathrm{CHs}$.

4) Transmission and scheduling in clusters: $\mathrm{A} \mathrm{CH}$ node schedules all sensor nodes in its cluster with TDMA (TimeDivision Multiple Access) medium access control to avoid collision.

5) Selecting transmission routes: Establish routes with routing algorithms for $\mathrm{CH}$ sensor nodes' data transmission, which concerns:

- First, minimize the hop number.

In step 2, the minimized hop number to the BS for each sensor node has been counted by broadcasting. So, we select the transmission routes with smallest hop number $H_{\text {route }}$.

$$
H_{\text {route }}=\operatorname{Min}\left\{H_{i}\right\}
$$

where $H_{i}$ is the hop number of route $i$ from all transmission routes from a sensor node to the BS.

- Second, maximize the energy in selected routes.

The method is that, calculate the lowest energy node in all routes from the selected routes. Then, select the route with the highest value of the lowest energy node. The route with the largest $E_{\text {route }}$ would be the route for transmission.

$$
E_{\text {route }}=\operatorname{Max}\left\{\operatorname{Min}\left\{E_{i j}^{\prime}\right\}\right\}
$$

where $E_{i j}^{\prime}$ is the energy of the lowest energy node $j$ in route $i$.

- Third, establish routes for transmission with the method addressed above, for all $\mathrm{CH}$ nodes and non-clustered sensor nodes, as the result in Figure 3

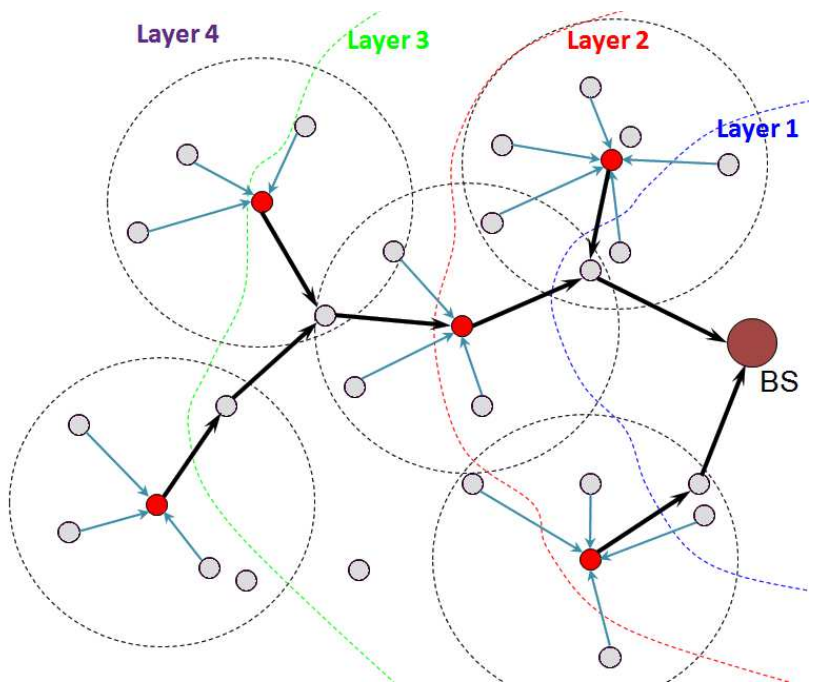

Figure 3. Routing for data transmission

- Last, discover the alternative sensor node (ASN) while transmitting data.

Here, we try to find if there exists an ASN $n\left(N_{\text {alt }}\right)$ for the sensor node $m$ near the transmission route. If so, the sensor node $m$ stops its routing function and let node $n$ take charge in the route.

$$
N_{\text {alt }}=\left\{\text { exist } \mid\left(S_{m n}<\frac{R}{2}\right) \bigcap\left(E_{n}>E_{m}\right)\right\} \ldots
$$

where $S_{m n}$ is the distance between two sensor nodes, $E$ is the energy of a sensor node, and $R$ is the transmission range for a sensor node.

\section{Evaluation AND ANALYsis}

In this section, we present some results obtained by simulation. We use the network simulator OMNeT++ |16] with $\mathrm{C}++$ programming to simulate the proposed algorithm, which is an open-architecture environment with GUI support.

In order to measure the proposed routing algorithm, we consider three metrics for the performance evaluation. System lifetime (the time of FND (first node dies)) and the number of alive nodes are applied, because lengthening the network lifetime is an important issue in clutered WSNs. The time of energy hole occurrence is applied, because we use ASN mechanism in routing for multi-hop transmissions.

- System lifetime (the time of FND) - The definition of system lifetime can be used to determine how alive a system is. There exist many lifetime definitions, such as the time when the first node dies (FND), the time when half of the nodes die, or the time that the network breaks in two or more segments $|3|$. We use the most general definition of the lifetime in this paper, the time of FND. Therefore, in order to maximize the system lifetime, we maximize the time of FND in a WSN system.

- The number of alive nodes - To keep the ability of sensing and gathering data in a WSN, it is essential to find how many sensor nodes are still functional in the entire system. The ability of a WSN depends on the set of alive nodes (nodes that have not failed). Therefore, we evaluate the functionality of the WSN system depending on counting the number of alive nodes in the system.

- The time of energy hole occurrence - The energy hole (self-induced black hole) problem around the BS appears, when the intermediary nodes near the BS died eariler than the sensor ndoes far away from the BS for the multi-hop transmissions. The time of energy hole occurrence indicates the time when the BS stop receiving data from sensor nodes. Moreover, the time of energy hole occurrence denotes the time length, in which the efficiency and integrity of data aggregation is ensured in the network by the time point, because the BS can no longer receive the data information if there is no intermediary node for multi-hop transmissions.

For the performance evaluation, we compare the proposed algorithm with three existing algorithms, called simple directed diffusion algorithm, non-clustering routing algorithm and $\mathrm{CH}$-based hierarchical algorithm, which are typical for 
the routing in WSNs with multi-hop transmissions. The three algorithms are described below.

1) The simple directed diffusion algorithm is an improvement of the classical flat-based routing, directed diffusion [17|. In this algorithm, each node runs a start-up routine, then starts the transmission. If the radio channel is busy, the node backs off; otherwise, the node transmits its data to the next-hop node. The sensor nodes which act as intermediaries relay received data only from the higher layers to the lower layers or the BS, in which way data are transmitted and directed to the BS.

2) The non-clustering routing algorithm is a routing algorithm without clustering. In this algorithm, each node runs the same process in data forwarding as the proposed routing algorithm without clustering process, which means each node runs a start-up routine, then only uses the round changing mechanism and routing algorithm for data transmission.

3) The CH-based hierarchical algorithm is a clusterbased hierarchical routing algorithm $[10 \mid$, in which the network is broken into layers. The sensed data travels from a $\mathrm{CH}$ in lower layer to a $\mathrm{CH}$ in higher layer, in turn the data is sent to the BS. To implement this algorithm, the routing algorithm is changed into the way that, the $\mathrm{CH}$ only selects the routes where there exits $\mathrm{CH}$ sensor nodes on the routes, then fix the route until the end of the current round.

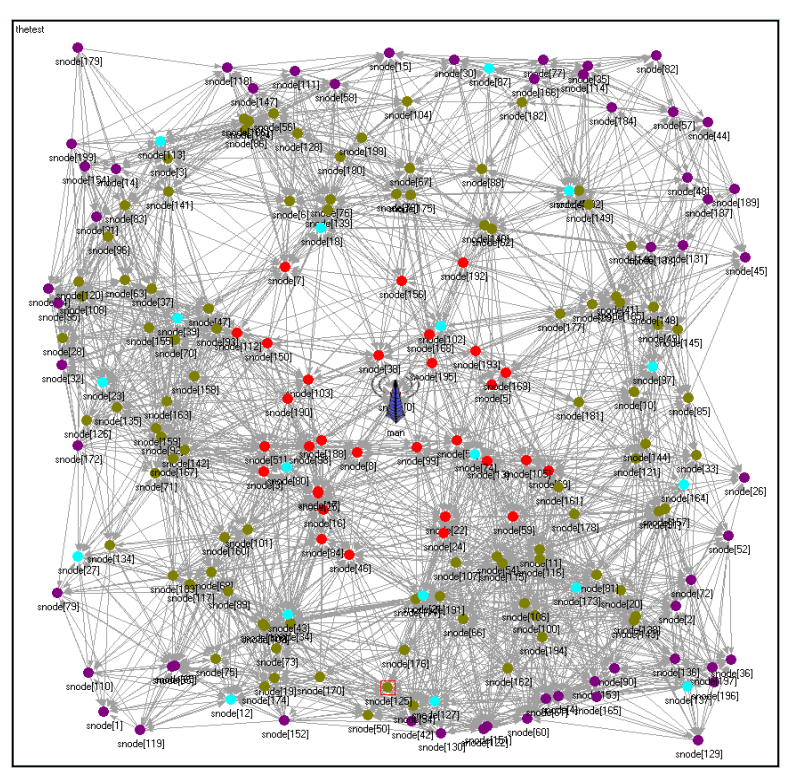

Figure 4. An illustration of simulation topology

In the simulation experiments, we use a network where 200 nodes were randomly distributed in a $400 m \times 400 m$ area, with a fixed BS located in the center of the sensing field, as shown in Figure 4. The transmission range of the BS and sensor nodes is $100 \mathrm{~m}$, and all sensor nodes periodically sense events and transmit the data packet to the BS. The initial energy of each sensor node is $0.5 \mathrm{~J}$, and the BS has unlimited energy. each data message is 512 bytes long, and the packet header for each type of packet is 25 bytes long. The communication energy parameter, electronics energy is set as: $E_{\text {elec }}=50 \mathrm{~nJ} / \mathrm{bit}$, and the energy for data aggregation is set as: $E_{\text {aggr }}=5 n J / b i t$.

Here, we first find a proper desired percentage of $\mathrm{CH}$ nodes $P$ during one round. Thus, in the first simulation, $P$ is set as $5 \%, 10 \%, 15 \%$, which aims at 5 percent, 10 percent, and 15 percent nodes for $\mathrm{CHs}$ per round. The optimum $P$ is expectated to consume lowest system energy dissipation. The sumulation result shows that, $P=10 \%$ is a better percentage of $\mathrm{CH}$ nodes, as shown in Figure 5, and we use this in the following simulations.

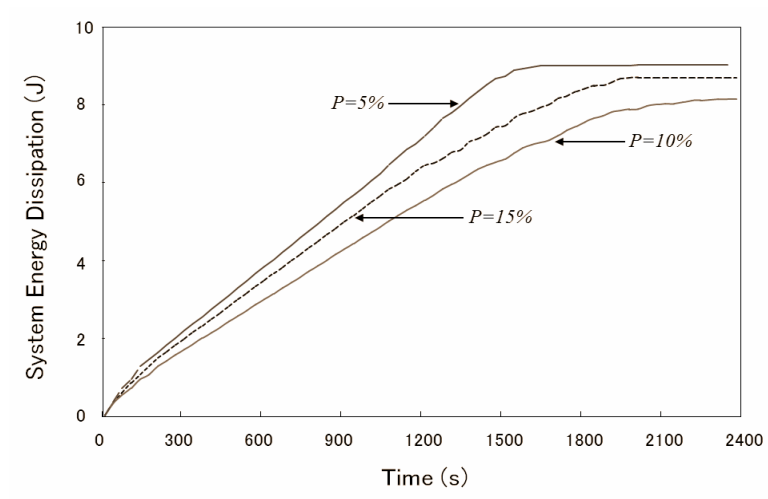

Figure 5. Finding the optimum $P$ with system energy dissipation

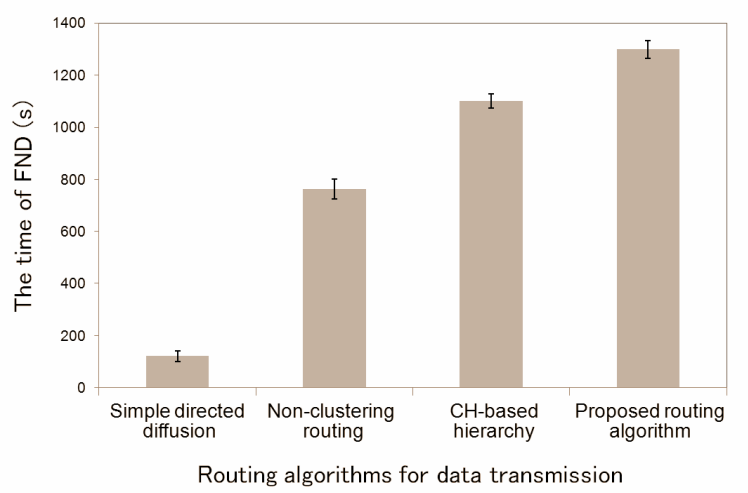

Figure 6. The time of FND in different algorithms

Figure 6 presents the time when the first node dies (FND) in different data transmission algorithms, which also indicates the balance of energy consumption in the system. We apply confidence intervals to our simulation results, and the certain percentage (confidence level) there is set to $95 \%$. Figure 7 shows the comparison of system lifetime using the proposed algorithm versus other three algorithms which are introduced above. Except for the latter parts of the results, the simulation results demonstrate that the system lifetime of the proposed algorithm is generally longer than that of 
other three routing algorithms. However, because of the selfinduced black hole problem, from the figure we can see that, the result becomes into nearly straight line near the end of the simulations. The reason is that sensor nodes in certain part of the system (far away from the BS) could not transmit the data packets to the BS, since the intermediary nodes died already for multi-hop transmissions to the BS. Figure 8 denotes the time of energy hole occurrence around the BS in different data transmission algorithms. It shows that the energy hole (self-induced black hole) problem appears the latest in the proposed routing algorithm, which means that the proposed algorithm mitigates the negative effect of the self-induced black hole more than the other algorithms. Furthermore, we can see that the straight line caused by this problem shows up the latest and the lowest in figure 7 with the proposed algorithm. The results demonstrate that the proposed algorithm has a better energy consumption balance in the system than the other three, because the time of FND appears the latest in the proposed algorithm.

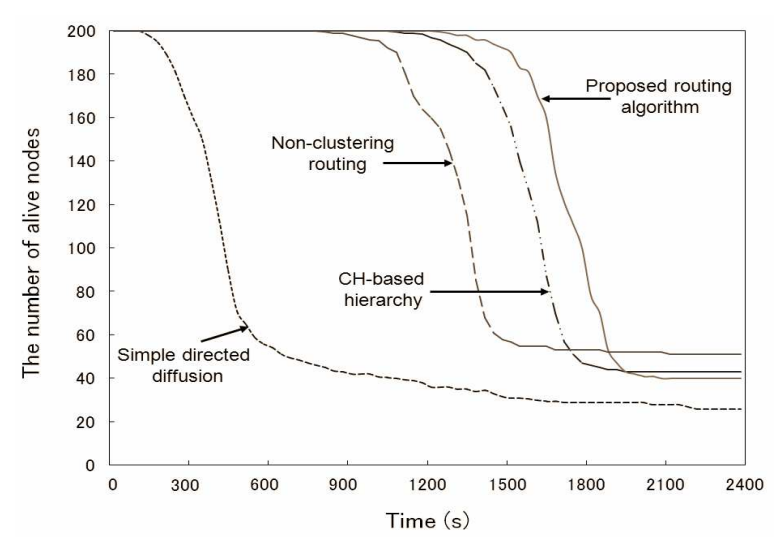

Figure 7. The number of alive nodes

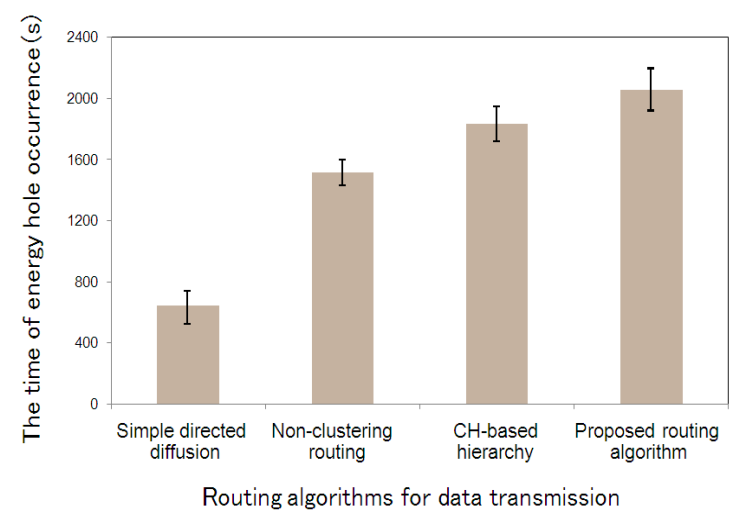

Figure 8. The time of energy hole occurrence in different algorithms

\section{CONCLusion ANd Future Work}

In this paper, we have introduced a novel power efficient routing algorithm for hierarchically clustered WSNs. The simulation results show that, compared with simple directional diffusion, non-clustering routing algorithm, and $\mathrm{CH}-$ based hierarchy, the proposed algorithm prolongs the system lifetime and mitigates the effect of self-induced black hole problem in large scale multi-hop WSNs.

The future work could include the improvements to simulation experiments with more variations for better results. Moreover, we are going to optimize the distance threshold used in the proposed ASN mechanism, because the threshold $R$ could also be conceived of $S=\frac{R}{3}$ or $S=\frac{2 R}{3}$. We would also consider more about the solution to solve the selfinduced black hole problem in the multi-hop transmissions.

\section{REFERENCES}

[1] K. Romer and F. Mattern, "The design space of wireless sensor networks," IEEE Wireless Communications, vol. 11, no. 6, pp. 54-61, 2004.

[2] "Definition of frequency bands." url: http://www.vlf.it/

[3] I. Akyildiz, W. Su, Y. Sankarasubramaniam, and E. Cayirci, "Wireless sensor networks: a survey," Computer Networks, vol. 38, no. 4, pp. 393-422, 2002.

[4] A. A. Abbasi and M. Younis, "A survey on clustering algorithms for wireless sensor networks," Computer Communications, vol. 30, no. 14-15, pp. 2826-2841, 2007.

[5] K. Akkaya and M. Younis, "A survey on routing protocols for wireless sensor networks," Ad Hoc Networks, vol. 3, no. 3, pp. 325-349, 2005.

[6] W. R. Heinzelman, A. Chandrakasan, and H. Balakrishnan, "Energy-efficient communication protocol for wireless microsensor networks," in Proc. of HICSS-33, 2000.

[7] S. Lindsey and C. S. Raghavendra, "PEGASIS: Powerefficient gathering in sensor information systems," in 2002 IEEE Aerospace Conf. Proc., vol. 3, 2002.

[8] J. Ibriq and I. Mahgoub, "Cluster-Based Routing in Wireless Sensor Networks: Issues and Challenges," in Proc. of SPECTS'04, 2004.

[9] C. Cano, B. Bellalta, P. Villalonga, and J. Perello, "Multihop cluster-based architecture for sparse wireless sensor networks," in Proc. of EW2008, 2008.

[10] Y. Jia, L. Zhao, and B. Ma, "A Hierarchical Clustering-based Routing Protocol for Wireless Sensor Networks Supporting Multiple Data Aggregation Qualities," IEEE Trans. on Parallel and Distributed Systems, vol. 4, no. 1-2, pp. 79-91, 2008.

[11] N. Ahmed, S. S. Kanhere, and S. Jha, "The holes problem in wireless sensor networks: a survey," ACM SIGMOBILE Mobile Computing and Com. Review, vol. 9, pp. 4-18, 2005.

[12] J. Li and P. Mohapatra, "Analytical modeling and mitigation techniques for the energy hole problem in sensor networks," Pervasive and Mobile Computing, vol. 3, no. 3, pp. 233-254, 2007.

[13] X. Wu, G. Chen, and S. K. Das, "Avoiding Energy Holes in Wireless Sensor Networks with Nonuniform Node Distribution," IEEE Trans. on Parallel and Distributed Systems, vol. 19, no. 5, pp. 710-720, 2008.

[14] A.-F. Liu, M. Ma, Z.-G. Chen, and W. hua Gui, "EnergyHole Avoidance Routing Algorithm for WSN," in Proc. of ICNC'08, 2008.

[15] M. Younis, M. Youssef, and K. Arisha, "Energy-aware routing in cluster-based sensor networks," in Proc. of MASCOTS2002, 2002.

[16] “OMNeT++ Community.” url: http://www.omnetpp.org/

[17] C. Intanagonwiwat, R. Govindan, and D. Estrin, "Directed diffusion: a scalable and robust communication paradigm for sensor networks," in Proc. of MobiCom'00, 2000. 